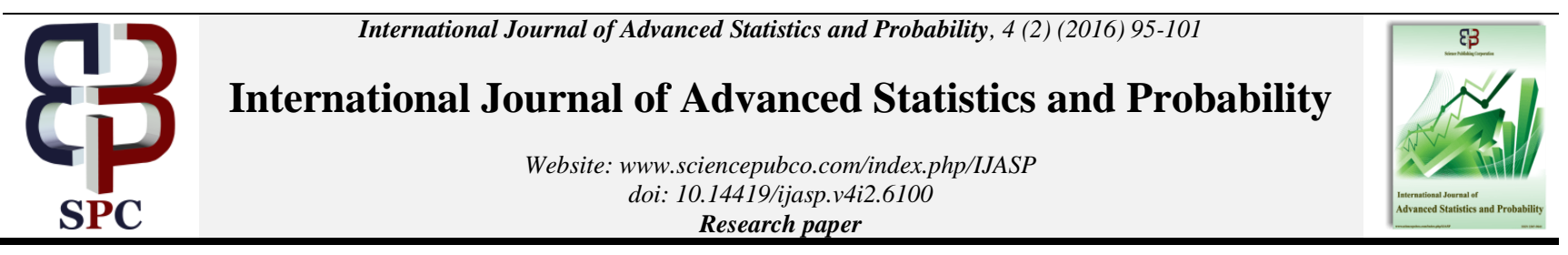

\title{
Transmutation of the two parameters Rayleigh distribution
}

\author{
Ehsan Ullah *, Mirza Naveed Shahzad \\ Department of Statistics, University of Gujrat, Gujrat, Pakistan \\ *Corresponding author E-mail: Ehsan.uog20@gmail.com
}

\begin{abstract}
In this study, transmuted two parameters Rayleigh distribution is proposed using quadratic rank transmutation map. This proposed distribution is more flexible and versatile than two parameters Rayleigh distribution. Some properties of the proposed distribution are derived such as moments, moment generating function, mean, variance, median, quantile function, reliability, and hazard function. The parameter estimation is approached through the method of least square estimation. The $r$ th and joint order statistics are also derived for the proposed distribution. The application of proposed model illustrated and compared using real data.
\end{abstract}

Keywords: Least Square Estimation; Moments; Order Statistics; Transmuted Two Parameters Rayleigh Distribution.

\section{Introduction}

Rayleigh distribution is widely used for survival analysis. Therefore, its application appeared in the medical sciences (patients survival times after operation, period of recurrence after surgery), engineering (component life cycles), computer sciences (failure rates of any software system), marketing (lifetimes of market rates of components) and in social sciences (duration that graduate person remain unemployed). For more details about lifetime applications, we refer Lai [10].

Lord Rayleigh (1880) introduced the single scale parameter Rayleigh distribution. It became much popular in survival analysis as it has hazard rate function that is an increasing function. Two parameters Rayleigh distribution with location and scale parameters also present in literature and recently, Dey et al. [2] used the two parameters Rayleigh distribution. Some more properties of two parameters Rayleigh distribution are derived by Leemis and McQueston [6] and Khan et al. [5] . Abd-Elfattah et al. [1] studied Bayes estimator and Dyer and Whisenand [3] introduced best linear unbiased estimator of Rayleigh distribution.

Merovci [7] introduced the transmuted Rayleigh distribution that has the scale and shape parameters and it proposed by using quadratic rank transmutation map (QRTM). In this study, the transmuted two parameters Rayleigh distribution is introduced with location, scale and shape parameters.

Rest of the paper is organized as follows. In section 2, the probability density function (pdf) and cumulative distribution function (cdf) of the transmuted two parameters Rayleigh distribution are derived and also graphically presented. The statistical properties, $r$ th moment, moments about the origin, mean, variance and moment generating function are derived in Section 3. Section 4 provides quantile function, random number generation and median of transmuted two parameters Rayleigh distribution. Section 5 is about hazard function and reliability function with their graphical presentation. Section 6 contains smallest, largest, $r$ th and joint order distribution of the proposed distribution. Parameters estimation of the distribution is discussed in Section 7. To compare the transmuted two parameters Rayleigh distribution with its parent distribution a real data set is used in Section 8.

\section{Transmuted two parameters Rayleigh dis- tribution}

A random variable $X$ has a transmuted distribution if its satisfy the relationship that is given by Shaw and Buckley [8] which is known as quadratic rank transmutation map(QRTM)

$F(x)=G(x)[(1+\lambda)-\lambda G(x)], \quad|\lambda| \leq 1$

Which on differentiation yields

$f(x)=g(x)[1+\lambda-2 \lambda G(x)]$

Where $\lambda$ is an additional parameter which is known as transmuted parameter and $g(x)$ and $G(x)$ are the pdf and cdf of base distribution.

The pdf of the two parameters Rayleigh distribution with location and scale parameters is

$g(x ; \alpha, \beta)=2 \beta(x-\alpha) e^{-\beta(x-\alpha)^{2}} \quad x>\alpha, \beta>0$

Where $\beta$ and $\alpha$ are scale and location parameters respectively.

And the corresponding cdf is defined as

$G(x ; \alpha, \beta)=1-e^{-\beta(x-\alpha)^{2}} \quad x>\alpha$

By using (2.1) and (2.4), we obtained the cdf of the transmuted two parameters Rayleigh distribution

$F_{T R}(x ; \alpha, \beta, \lambda)=\left[1-e^{-\beta(x-\alpha)^{2}}\right]\left[1+\lambda e^{-\beta(x-\alpha)^{2}}\right]$

And by using (2.2),(2.3) and (2.4) or by differentiation (2.5) gives the corresponding pdf of transmuted two parameters Rayleigh distribution that is defined as

$f_{T R}(x ; \alpha, \beta, \lambda)=2 \beta(x-\alpha) e^{-\beta(x-\alpha)^{2}}\left[1-\lambda+2 \lambda e^{-\beta(x-\alpha)^{2}}\right]$

The transmuted parameter $\lambda$ has range -1 to +1 and if we take $\lambda=0$ then this transmuted pdf and cdf reduces to the base distri- 
bution. Various shapes of the cdf's and pdf's with different values of parameters are illustrated in Fig. 1 and Fig. 2 respectively.
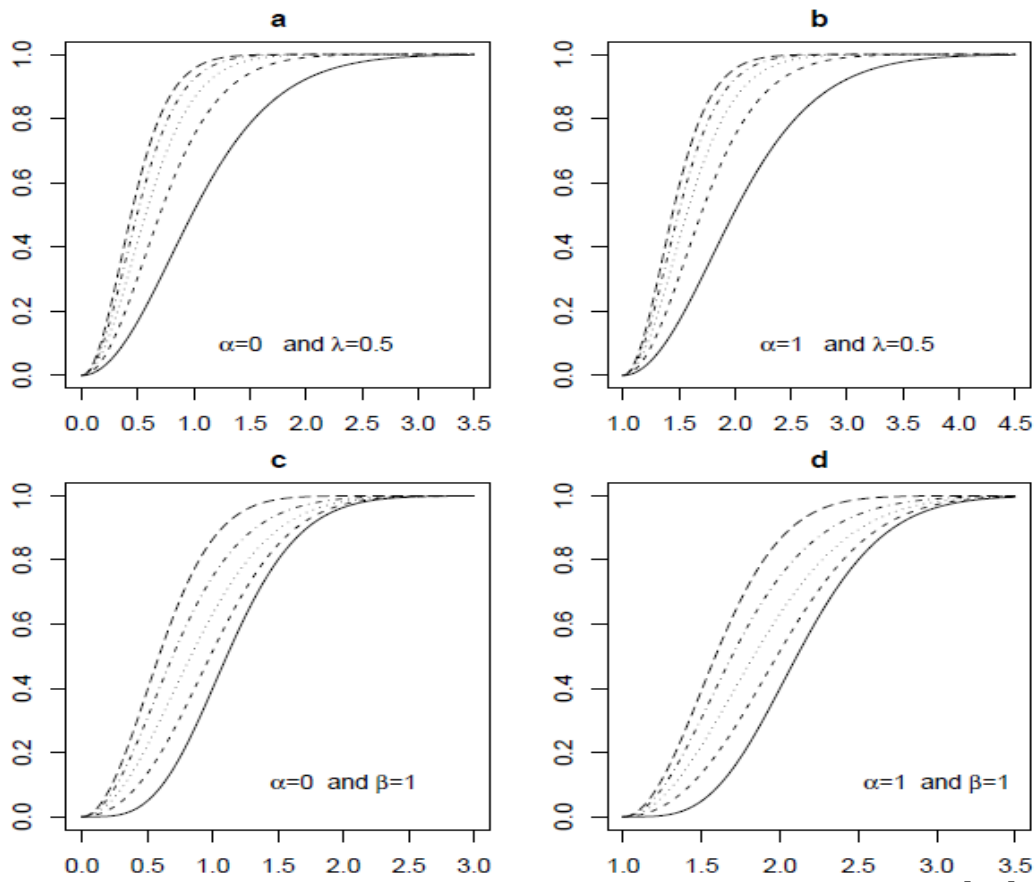

Fig. 1: The cdf's of various Transmuted two parameters Rayleigh Distribution for values of parameters: a) $\beta=0.5[0.5] 2.5$, b) $\beta=0.5[0.5] 2.5$, c) $\lambda=-1[0.5] 1$, D) $\lambda=-1[0.5] 1$ with Solid, Dashed, Dotted, Dotdash and Longdash lines respectively.

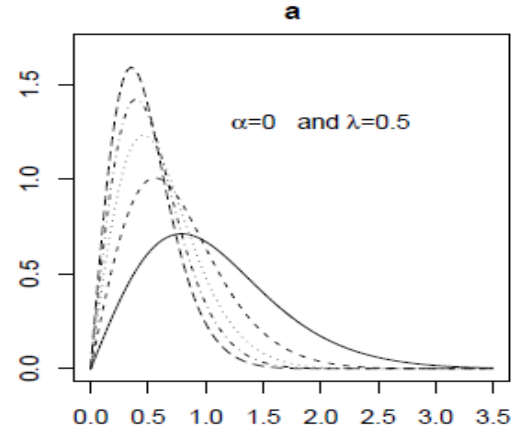

c

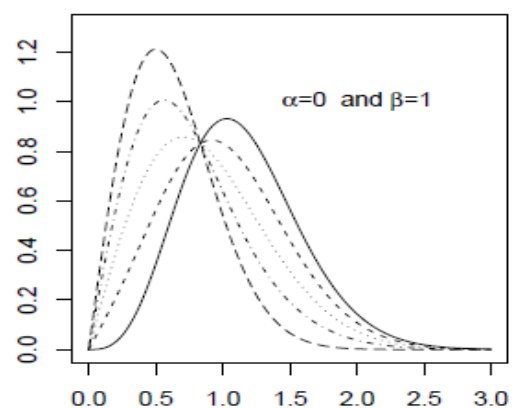

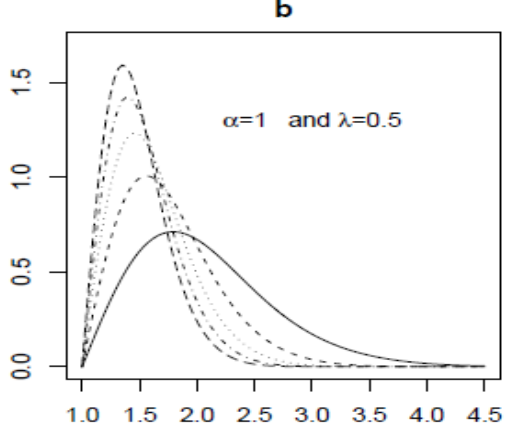

d

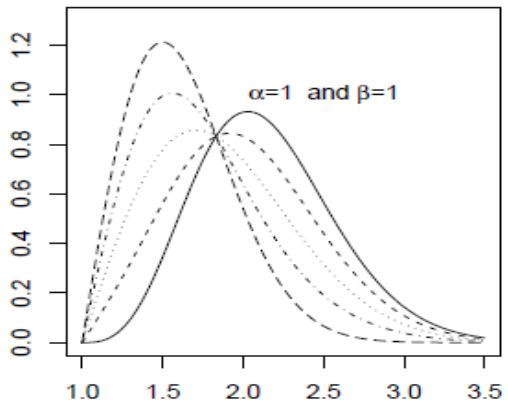

Fig. 2: The pdf's of Various Transmuted two parameters Rayleigh distribution for values of parameters: $\alpha$ ) $\beta=0.5[0.5] 2.5$, b) $\beta=0.5[0.5] 2.5$, c) $\lambda=-1[0.5] 1$, d) $\lambda=-1[0.5] 1$ with Solid, Dashed, Dotted, Dotdash and Longdash lines respectively.

\section{Moments}

In this section, some statistical properties of transmuted two parameters Rayleigh distribution such as moments, mean, variance, the area under the curve and moment generating function are derived and discussed.

Theorem 3.1: Let $X$ is a random variable that has $T_{R}(x ; \beta, \alpha, \lambda)$ with $|\lambda| \leq 1$, then rth moment $E\left(x^{r}\right)$ of transmuted two parameters Rayleigh distribution is

$$
\mu_{r}^{\prime}=\sum_{k=0}^{r}\left(\begin{array}{l}
r \\
k
\end{array}\right) \alpha^{r-k}\left[(1-\lambda)\left\{\frac{\Gamma\left(\frac{k}{2}+1\right)}{\beta^{\left(\frac{k}{2}\right)}}\right\}+\lambda\left\{\frac{\Gamma\left(\frac{k}{2}+1\right)}{(2 \beta)^{\left(\frac{k}{2}\right)}}\right\}\right]
$$

Proof: The $r$ th moment is given by

$$
\begin{gathered}
\mu_{r}^{\prime}=E\left(X^{r}\right)=\int_{\alpha}^{\infty} x^{r} 2 \beta(x-\alpha) e^{-\beta(x-\alpha)^{2}}[(1-\lambda) \\
\left.+2 \lambda e^{-\beta(x-\alpha)^{2}}\right] d x
\end{gathered}
$$

For convenience substitute $x=\alpha+z$, then 
$\mu_{r}^{\prime}=2 \beta \int_{0}^{\infty}(\alpha+z)^{r} z e^{-\beta z^{2}}\left[(1-\lambda)+2 \lambda e^{-\beta z^{2}}\right] d z$

$\mu_{r}^{\prime}=2 \beta \int_{0}^{\infty} \sum_{k=0}^{r}\left(\begin{array}{l}r \\ k\end{array}\right) z^{k} \alpha^{r-k} z e^{-\beta z^{2}}\left[(1-\lambda)+2 \lambda e^{-\beta z^{2}}\right] d z$

$\mu_{r}^{\prime}=2 \beta \sum_{k=0}^{r}\left(\begin{array}{l}r \\ k\end{array}\right) \alpha^{r-k} \int_{0}^{\infty} z^{k+1} e^{-\beta z^{2}}\left[(1-\lambda)+2 \lambda e^{-\beta z^{2}}\right] d z$

Again for convenience we substitute $y=z^{2}$, then

$$
\begin{aligned}
\mu_{r}^{\prime}=2 \beta \sum_{k=0}^{r}\left(\begin{array}{l}
r \\
k
\end{array}\right) \alpha^{r-k} & {\left[(1-\lambda) \int_{0}^{\infty} \frac{y^{\left(\frac{k+1}{2}\right)} e^{-\beta y}}{2 \sqrt{y}} d y\right.} \\
+ & \left.2 \lambda \int_{0}^{\infty} \frac{y^{\left(\frac{k+1}{2}\right)} e^{-2 \beta y}}{2 \sqrt{y}} d y\right]
\end{aligned}
$$

$\mu_{r}^{\prime}=2 \beta \sum_{k=0}^{r}\left(\begin{array}{l}r \\ k\end{array}\right) \alpha^{r-k}\left[\frac{(1-\lambda)}{2} \int_{0}^{\infty} y^{\left(\frac{k}{2}\right)} e^{-\beta y} d y\right.$

$$
\left.+\lambda \int_{0}^{\infty} y^{\left(\frac{k}{2}\right)} e^{-2 \beta y} d y\right]
$$

$\mu_{r}^{\prime}=\sum_{k=0}^{r}\left(\begin{array}{l}r \\ k\end{array}\right) \alpha^{r-k}\left[(1-\lambda)\left\{\frac{\Gamma\left(\frac{k}{2}+1\right)}{\beta^{\left(\frac{k}{2}\right)}}\right\}+\lambda\left\{\frac{\Gamma\left(\frac{k}{2}+1\right)}{(2 \beta)^{\left(\frac{k}{2}\right)}}\right\}\right]$

By setting $r=1$ and $r=2$ in (3.1), we obtain mean, $1^{\text {st }}$ and $2^{\text {nd }}$ moments. The mean of transmuted two parameters Rayleigh distribution by using (3.1) can be defined as

Mean $=E(X)=\alpha+\frac{\sqrt{\pi}}{2 \beta}\left[(1-\lambda)+\frac{\lambda}{\sqrt{2}}\right]$

By using $r$ th moment expression, $1^{\text {st }}$ and $2^{\text {nd }}$ moments about origin of transmuted Rayleigh distribution may be defined as

The $1^{\text {st }}$ moment is given, by setting $r=1$ in (3.1)

$\mu_{1}^{\prime}=\alpha+\frac{\sqrt{\pi}}{2 \sqrt{\beta}}\left[(1-\lambda)+\frac{\lambda}{\sqrt{2}}\right]$

The $2^{\text {nd }}$ moment about origin is given by setting $r=2$ in (3.1)

$\mu_{2}^{\prime}=\alpha^{2}+\frac{\alpha \sqrt{\pi}}{\sqrt{\beta}}\left[(1-\lambda)+\frac{\lambda}{\sqrt{2}}\right]+\frac{1}{\beta}\left[(1-\lambda)+\frac{\lambda}{2}\right]$

Coefficient of variation $(C V)$, Skewness $(S k)$, and Kurtosis $(K r)$ of transmuted two parameters Rayleigh distribution can be derived by using following expressions through $\alpha_{r}^{\prime}(r=1,2,3,4)$

If $\mu_{2}=\mu_{2}^{\prime}-\left(\mu_{1}^{\prime}\right)^{2}$

$\mu_{3}=\mu_{3}^{\prime}-3 \mu_{2}^{\prime} \mu_{1}^{\prime}+2\left(\mu_{1}^{\prime}\right)^{3}$

$\mu_{4}=\mu_{4}^{\prime}-4 \mu_{3}^{\prime} \mu_{1}^{\prime}+6 \mu_{2}^{\prime} \mu_{1}^{\prime 2}+2\left(\mu_{1}^{\prime}\right)^{3}$

Then

$$
C V=\frac{\sigma}{\mu_{1}^{\prime}}
$$

$S k=\frac{\mu_{3}^{\prime}-3 \mu_{2}^{\prime} \mu_{1}^{\prime}+2\left(\mu_{1}^{\prime}\right)^{3}}{\sigma^{3}}$

$K r=\beta_{2}=\frac{\mu_{4}^{\prime}-4 \mu_{3}^{\prime} \mu_{1}^{\prime}+6 \mu_{2}^{\prime} \mu_{1}^{\prime 2}+2\left(\mu_{1}^{\prime}\right)^{3}}{\sigma^{4}}$

Theorem 3.2: Let the random variable $X$ follows transmuted two parameters Rayleigh distribution, and then its variance has the following form
$\sigma^{2}=\operatorname{Var}(X)=\frac{1}{\beta}\left[(1-\lambda)+\frac{\lambda}{2}\right]-\frac{\pi}{4 \beta}\left[(1-\lambda)+\frac{\lambda}{2}\right]^{2}$

Proof. The Variance of transmuted two parameters Rayleigh distribution is given as

$\operatorname{Var}(X)=E\left(X^{2}\right)-[E(X)]^{2}$

Then

$$
\begin{gathered}
\operatorname{Var}(X)=\alpha^{2}+\frac{\alpha \sqrt{\pi}}{\sqrt{\beta}}\left[(1-\lambda)+\frac{\lambda}{\sqrt{2}}\right]+\frac{1}{\beta}\left[(1-\lambda)+\frac{\lambda}{2}\right] \\
-\left[\alpha+\frac{\sqrt{\pi}}{2 \sqrt{\beta}}\left[(1-\lambda)+\frac{\lambda}{\sqrt{2}}\right]\right]^{2} \\
\operatorname{Var}(X)=\alpha^{2}+\frac{\alpha \sqrt{\pi}}{\sqrt{\beta}}\left[(1-\lambda)+\frac{\lambda}{\sqrt{2}}\right]+\frac{1}{\beta}\left[(1-\lambda)+\frac{\lambda}{2}\right]-\alpha^{2} \\
-\frac{\pi}{4 \beta}\left[(1-\lambda)+\frac{\lambda}{2}\right]^{2}-\frac{\alpha \sqrt{\pi}}{\sqrt{\beta}}\left[(1-\lambda)+\frac{\lambda}{\sqrt{2}}\right] \\
\operatorname{Var}(X)=\frac{1}{\beta}\left[(1-\lambda)+\frac{\lambda}{2}\right]-\frac{\pi}{4 \beta}\left[(1-\lambda)+\frac{\lambda}{2}\right]^{2}
\end{gathered}
$$

Theorem 3.3: Let $X$ has a transmuted two parameters Rayleigh distribution, and then the moment generating function (mgf) of transmuted two parameters Rayleigh distribution is

$M_{X}(t)=\sum_{r=\alpha}^{\infty} \sum_{k=0}^{r} \frac{t^{r}}{r !}\left(\begin{array}{l}r \\ k\end{array}\right) \alpha^{r-k}\left[\begin{array}{c}(1-\lambda)\left\{\frac{\Gamma\left(\frac{k}{2}+1\right)}{\beta^{\left(\frac{k}{2}\right)}}\right\} \\ +\lambda\left\{\frac{\Gamma\left(\frac{k}{2}+1\right)}{(2 \beta)^{\left(\frac{k}{2}\right)}}\right\}\end{array}\right]$

Proof: The mgf for $X$ is given as

$M_{X}(t)=E\left(e^{t X}\right)=\int_{\alpha}^{\infty} e^{t x} f_{T R}(x ; \alpha, \beta, \lambda) d x$

We know that

$e^{t x}=1+t x+\frac{t^{2} x^{2}}{2 !}+\frac{t^{3} x^{3}}{3 !}+\cdots \frac{t^{r} x^{r}}{r !}+\cdots$

Then

$$
\begin{aligned}
& M_{X}(t)=\int_{\alpha}^{\infty}\left(1+t x+\frac{t^{2} x^{2}}{2 !}+\frac{t^{3} x^{3}}{3 !}\right. \\
& \left.+\cdots \frac{t^{n} x^{n}}{n !}+\ldots \ldots\right) f_{T R}(x ; \alpha, \beta, \lambda) d x \\
& M_{X}(t)=\sum_{r=\alpha}^{\infty} \frac{t^{r} E(X)^{r}}{r !} \\
& M_{X}(t)=\sum_{r=\alpha}^{\infty} \frac{t^{r}}{r !}\left(\sum _ { k = 0 } ^ { r } ( \begin{array} { l } 
{ r } \\
{ k }
\end{array} ) \alpha ^ { r - k } \left[(1-\lambda)\left\{\frac{\Gamma\left(\frac{k}{2}+1\right)}{\beta^{\left(\frac{k}{2}\right)}}\right\}\right.\right. \\
& \left.\left.+\lambda\left\{\frac{\Gamma\left(\frac{k}{2}+1\right)}{(2 \beta)^{\left(\frac{k}{2}\right)}}\right\}\right]\right)
\end{aligned}
$$




$$
\begin{gathered}
M_{X}(t)=\sum_{r=\alpha}^{\infty} \sum_{k=0}^{r} \frac{t^{r}}{r !}\left(\begin{array}{l}
r \\
k
\end{array}\right) \alpha^{r-k}\left[( 1 - \lambda ) \left\{\frac{\Gamma\left(\frac{k}{2}+1\right)}{\left.\beta^{\left(\frac{k}{2}\right)}\right\}}\right.\right. \\
\left.+\lambda\left\{\frac{\Gamma\left(\frac{k}{2}+1\right)}{(2 \beta)^{\left(\frac{k}{2}\right)}}\right\}\right]
\end{gathered}
$$

Theorem 3.4: Suppose $X$ has a transmuted two parameters Rayleigh distribution, then total area of transmuted two parameters Rayleigh distribution under the curve is unity.

Area $=\int_{\alpha}^{\infty} 2 \beta(x-\alpha) e^{-\beta(x-\alpha)^{2}}\left[1-\lambda+2 \lambda e^{-\beta(x-\alpha)^{2}}\right] d x=1$

Proof: The area under the curve is

Area $=\int_{\alpha}^{\infty} 2 \beta(x-\alpha) e^{-\beta(x-\alpha)^{2}}\left[1-\lambda+2 \lambda e^{-\beta(x-\alpha)^{2}}\right] d x$

For convenience we substitute $z=x-\alpha$, then

Area $=2 \beta \int_{0}^{\infty} z e^{-\beta z^{2}}\left[(1-\lambda)+2 \lambda e^{-\beta z^{2}}\right] d z$

Area $=2 \beta(1-\lambda) \int_{0}^{\infty} z e^{-\beta z^{2}}+4 \beta \lambda \int_{0}^{\infty} z e^{-2 \beta z^{2}} d z$

Again for convenience we substitute $z^{2}=y$, then

$$
\begin{aligned}
& \begin{aligned}
\text { Area }=2 \beta(1-\lambda) & \int_{0}^{\infty} \sqrt{y} e^{-\beta y} \frac{1}{2 \sqrt{y}} d y \\
& +4 \beta \lambda \int_{0}^{\infty} \sqrt{y} e^{-2 \beta y} \frac{1}{2 \sqrt{y}} d y
\end{aligned} \\
& \text { Area }=(1-\lambda) \beta\left|\frac{e^{-\beta y}}{-\beta}\right|_{0}^{\infty}+2 \lambda \beta\left|\frac{e^{-2 \beta y}}{-2 \beta}\right|_{0}^{\infty} \\
& \text { Area }=-(1-\lambda)\left[e^{-\infty}-e^{0}\right]-\lambda\left[e^{-\infty}-e^{0}\right] \\
& \text { Area }=(1-\lambda)+\lambda=1
\end{aligned}
$$

\section{Quantile function Random number genera- tion and Median}

Hyndman and Fan [4] introduced the samples quantile function for every distribution in this shape

$$
Q(q)=F^{-1}(q)=\inf \{x: F(x) \geq q\} \quad 0<q<1
$$

Where $F(x)$ is the distribution function. Quantile function divides the ordered data into $q$ th equal parts. The $q$ th quantile function of transmuted Rayleigh distribution by using (4.1) and (2.5) is given as
$Q(q)=x$
$=\alpha+\left[-\frac{1}{\beta} \log \left[\frac{(\lambda-1) \pm \sqrt{\lambda^{2}+1+2 \lambda-4 \lambda q}}{2 \lambda}\right]\right]^{\frac{1}{2}}$

The expression (4.2) can also be used for random number generation if we substitute $u$ rather than $q$, where $u$ follows standard uniform variate then random numbers are generated as

$$
x=\alpha+\left[-\frac{1}{\beta} \log \left[\frac{(\lambda-1) \pm \sqrt{\lambda^{2}+1+2 \lambda-4 \lambda u}}{2 \lambda}\right]\right]^{\frac{1}{2}}
$$

Median is $50^{\text {th }}$ percentiles, and then median of transmuted two parameters Rayleigh distribution by using (4.2) and if we substitute $q=0.5$, then we can find median of transmuted Rayleigh distribution

$x_{0.5}=\alpha+\left[-\frac{1}{\beta} \log \left[\frac{(\lambda-1) \pm \sqrt{\lambda^{2}+1}}{2 \lambda}\right]\right]^{\frac{1}{2}}$

\section{Reliability analysis}

The Reliability function $R(t)$ gives the failure of a system or gives the probability of surviving of an item at reach on $t$ time. The reliability function of transmuted two parameters Rayleigh distribution is given by

$$
\begin{gathered}
R_{T R}(t)=P(T>t)=\int_{t}^{\infty} f(t) d t=1-F_{T R}(t) \\
R_{T R}(t)=e^{-\beta(t-\alpha)^{2}}\left(1-\lambda\left(1-e^{-\beta(t-\alpha)^{2}}\right)\right)
\end{gathered}
$$

With different values of parameters, we can get different patterns of reliability function of transmuted two parameters Rayleigh distribution. Fig. 3 illustrates the pattern of reliability function of transmuted two parameters Rayleigh distribution.

An important property of life phenomenon in life testing analysis is hazard rate function. It measures the failure rate and it depends on time. If hazard rate value will increase then the probability of failure will also increase. Hazard rate function by using (2.6) and (5.1) can be defined as

$$
h(t)=\frac{f(t)}{R(t)}
$$

$x_{0.5}=\alpha+\left[-\frac{1}{\beta} \log \left[\frac{(\lambda-1) \pm \sqrt{\lambda^{2}+1}}{2 \lambda}\right]\right]^{\frac{1}{2}}$ 


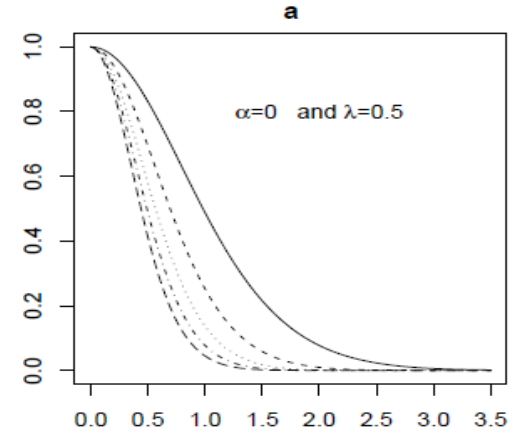

c

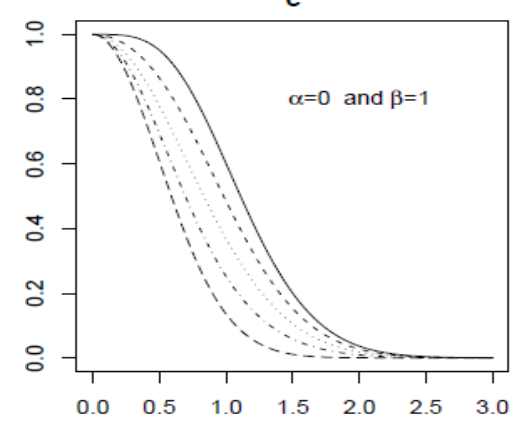

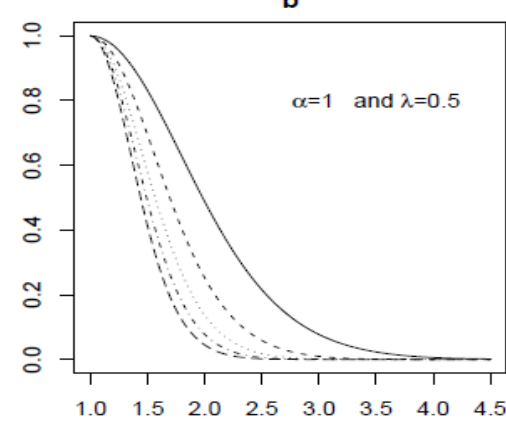

d

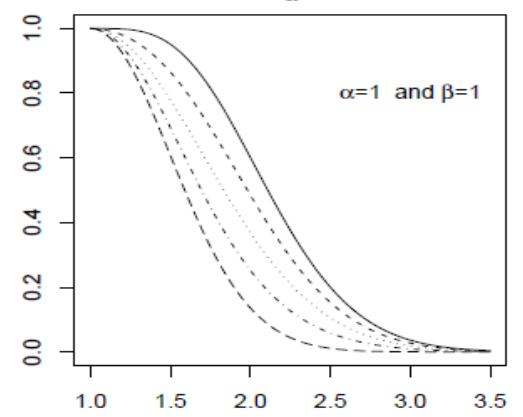

Fig. 3: The reliability function of various Transmuted two parameters Rayleigh distribution for values of parameters: $a$ ) $\beta=0.5[0.5] 2.5$, b) $\beta=0.5[0.5] 2.5$, c) $\lambda=-1[0.5] 1, d) \lambda=-1[0.5] 1$ with Solid, Dashed, Dotted, Dotdash and Longdash Lines Respectively.

\section{Order statistics of transmuted two parame- ters Rayleigh distribution}

The distribution of smallest, largest, $r$ th and joint order statistics are most important functions. Order statistics can be defined mathematically as, let $X_{1}, X_{2}, \ldots \ldots, X_{n}$ are random variables and can be ordered as $X_{(1)} \leq X_{(2)} \leq \ldots \ldots \leq X_{(n)}$ then the values $X_{(1)}, X_{(2)}, \ldots \ldots, X_{(n)}$ are ordered statistics of random variables.

The smallest order statistics of transmuted two parameters Rayleigh distribution is obtained as

$$
\begin{aligned}
& f_{(1)}\left(x_{(1)}\right)=n\left[1-F\left(x_{(1)}\right)\right]^{n-1} f\left(x_{(1)}\right) \\
& f_{(1)}\left(x_{(1)}\right)=2 n \beta\left(x_{(1)}-\alpha\right) e^{-\beta\left(x_{(1)}-\alpha\right)^{2}}\left[1-\lambda+2 \lambda e^{-\beta\left(x_{(1)}-\alpha\right)^{2}}\right] \\
& \times \sum_{i=0}^{n-1} \sum_{j=0}^{i} \sum_{k=0}^{i}\left(\begin{array}{c}
n-1 \\
i
\end{array}\right)\left(\begin{array}{l}
i \\
j
\end{array}\right)\left(\begin{array}{l}
i \\
k
\end{array}\right)(-1)^{i+j}(\lambda)^{k}\left[\left(e^{-\beta\left(x_{(1)}-\alpha\right)^{2}}\right)\right]^{j+k}
\end{aligned}
$$

Let suppose that maximum values follow the transmuted two parameters Rayleigh distribution, and then the distribution of $n$th or maximum order statistics can be defined as

$$
\begin{aligned}
f_{(n)}\left(x_{(n)}\right) & =n\left[F\left(x_{(n)}\right)\right]^{n-1} f\left(x_{(n)}\right) \\
f_{(n)}\left(x_{(n)}\right) & =2 n \beta\left[\left(x_{(n)}-\alpha\right) e^{-\beta\left(x_{(n)}-\alpha\right)^{2}}\left[1-\lambda+2 \lambda e^{-\beta\left(x_{(n)}-\alpha\right)^{2}}\right]\right] \\
\times & \sum_{i=0}^{n-1} \sum_{j=0}^{n-1}\left(\begin{array}{c}
n-1 \\
i
\end{array}\right)\left(\begin{array}{c}
n-1 \\
j
\end{array}\right)(-1)^{i}(\lambda)^{j}\left[e^{-(i+j) \beta\left(x_{(n)}-\alpha\right)^{2}}\right]
\end{aligned}
$$

$f_{(r)}\left(x_{(r)}\right)$

$=\frac{2 \beta n !}{(r-1) !(n-r) !}\left(x_{(r)}-\alpha\right) e^{-\beta\left(x_{(r)}-\alpha\right)^{2}}$

$\times\left[1-\lambda+2 \lambda e^{-\beta\left(x_{(r)}-\alpha\right)^{2}}\right]$

$\times \sum_{i=0}^{r-1} \sum_{j=0}^{r-1} \sum_{k=0}^{n-r} \sum_{l=0}^{k} \sum_{m=0}^{k}\left(\begin{array}{c}r-1 \\ i\end{array}\right)\left(\begin{array}{c}r-1 \\ j\end{array}\right)\left(\begin{array}{c}n-r \\ k\end{array}\right)\left(\begin{array}{l}k \\ l\end{array}\right)\left(\begin{array}{c}k \\ m\end{array}\right)$

$\times(-1)^{i+k+l}(\lambda)^{j+l} e^{-\beta(i+j+l+m)\left(x_{(r)}-\alpha\right)^{2}}$

The joint pdf of the transmuted two parameters Rayleigh distribution of $X_{(r)}$ and $X_{(s)}$, when $1 \leq r \leq s \leq n$ is defined as

$$
\begin{aligned}
& f\left(x_{(r)}, x_{(s)}\right)=\frac{n !}{(r-1) !(s-r-1)(n-s) !}\left[F\left(x_{r}\right)\right]^{r-1} f\left(x_{(r)}\right) \\
& \times\left[F\left(x_{(s)}\right)-F\left(x_{(r)}\right]^{s-r-1} f\left(x_{(s)}\right)\left[1-F\left(x_{(s)}\right)\right]^{n-s}\right. \\
& =\frac{4 \beta^{2} n !}{(r-1) !(s-r-1) !(n-s) !}\left(x_{(r)}-\alpha\right)\left(x_{(s)}-\alpha\right) e^{-\beta\left(x_{r}-\alpha\right)^{2}} \\
& \times e^{-\beta\left(x_{(s)}-\alpha\right)^{2}}\left[1-\lambda+2 \lambda e^{-\beta\left(x_{(r)}-\alpha\right)^{2}}\right]\left[1-\lambda+2 \lambda e^{-\beta\left(x_{(s)}-\alpha\right)^{2}}\right] \\
& \times \sum_{i=0}^{r-1} \sum_{j=0}^{r-1} \sum_{k=0}^{s-r-1} \sum_{l=0}^{n-s} \sum_{m=0}^{k} \sum_{n=0}^{k} \sum_{p=0}^{s+l-r-k-1} \sum_{q=0}^{s+l-r-k-1}\left(\begin{array}{c}
r-1 \\
i
\end{array}\right)\left(\begin{array}{c}
r-1 \\
j
\end{array}\right) \\
& \times\left(\begin{array}{c}
s-r-1 \\
k
\end{array}\right)\left(\begin{array}{c}
n-s \\
l
\end{array}\right)\left(\begin{array}{c}
k \\
m
\end{array}\right)\left(\begin{array}{l}
k \\
n
\end{array}\right)\left(\begin{array}{c}
s+l-r-k-1 \\
p
\end{array}\right) \\
& \times\left(\begin{array}{c}
s+l-r-k-1 \\
q
\end{array}\right)(-1)^{i+k+l+m+p}(\lambda)^{j+n+q} \\
& \times e^{-\beta(i+j+m+n)\left(x_{(r)}-\alpha\right)^{2}} e^{-\beta(p+q)\left(x_{(s)}-\alpha\right)^{2}}
\end{aligned}
$$

\section{Parameter estimation}

Generally, the $r$ th order statistics of transmuted two parameters Rayleigh distribution has specified pdf is given as

$f_{(r)}\left(x_{(r)}\right)=\frac{n !}{(r-1) !(n-r) !}\left[F\left(x_{(r)}\right)\right]^{r-1}\left[1-F\left(x_{(r)}\right)\right]^{n-r} f\left(x_{(r)}\right)$
In this section, parameters estimation of transmuted two parameters Rayleigh distribution is discussed. The parameters estimate integrated through the least square estimation method. Let $X_{1}, X_{2}, X_{3}, \ldots, X_{n}$ be a random sample of transmuted two parameters Rayleigh distribution with its $\operatorname{cdf} F_{T R}(x)$ and suppose $X_{i}$ denote the ordered sample where $i=1,2,3, \ldots n$. For $n$ sample size, we have rank as

$E\left(F\left(x_{i}\right)\right)=\frac{i}{n+1}$ 
The least square estimation (LSE) can be obtained by minimizing the expression

$Q(x ; \alpha, \beta, \lambda)=\sum_{i=0}^{n}\left(F\left(x_{i}\right)-\frac{i}{n+1}\right)^{2}$

Now putting (2.5) in (7.1) we can write

$$
Q(x ; \alpha, \beta, \lambda)=\sum_{i=0}^{n}\left[\begin{array}{c}
\left(1-e^{-\beta(x-\alpha)^{2}}\right)\left(1+\lambda e^{-\beta(x-\alpha)^{2}}\right) \\
-\frac{i}{n+1}
\end{array}\right]^{2}
$$

To obtain the parameters estimates the (7.2) differentiated with respect to $\alpha, \beta$ and $\lambda$. Finally the following expression are obtained

$$
\begin{aligned}
& \sum_{i=0}^{n}\left[\left(1-e^{-\beta(t-\alpha)^{2}}\right)\left(1+\lambda e^{-\beta(t-\alpha)^{2}}\right)-\frac{i}{n+1}\right] \\
& \times\left[2 \beta(x-\alpha) e^{-\beta(t-\alpha)^{2}}\left[1-\lambda\left(1+\lambda e^{-\beta(t-\alpha)^{2}}\right)\right]\right]=0 \\
& \sum_{i=0}^{n}\left[\left(1-e^{-\beta(t-\alpha)^{2}}\right)\left(1+\lambda e^{-\beta(t-\alpha)^{2}}\right)-\frac{i}{n+1}\right] \\
& \times(x-\alpha)^{2} e^{-\beta(t-\alpha)^{2}}\left[1-\lambda\left(1-2 e^{-\beta(t-\alpha)^{2}}\right)\right]=0 \\
& \sum_{i=0}^{n}\left[1+\lambda e^{-\beta(t-\alpha)^{2}}-e^{-\beta(t-\alpha)^{2}}-\lambda e^{-2 \beta(t-\alpha)^{2}}-\frac{i}{n+1}\right] \\
& \times e^{-\beta(t-\alpha)^{2}}\left(1-e^{-2 \beta(t-\alpha)^{2}}\right)=0
\end{aligned}
$$

The perfect solution of the estimator for unknown parameters is not possible and then the estimates of parameters are derived by solving above three nonlinear equations simultaneously. This can be done by Newton-Raphson approach to numerically maximize the log likelihood function.

\section{Applications}

In this section, an empirical study is performed using real data set to compare the fitting of the transmuted two parameters Rayleigh distribution with its parent distributions. This comparison is evaluated on the basis of Akaike Information Criterion (AIC), Bayesian Information Criterion (BIC) and Consistent Akaike Information Criterion used to check the fitting of the distributions.

The data set consists of the precipitation level (inches) which was recorded in Minneapolis-St. Paul area during the month of March over 30 years period. The length of this data set is based on 30 observations and given in Tierney [9].

Table 1: The Summary Statistics of Precipitation Level (Inches) During the Month of March.

\begin{tabular}{|l|l|}
\hline Length & 30 \\
\hline Average & 1.6750 \\
\hline Minimum & 0.3200 \\
\hline Maximum & 4.7500 \\
\hline$Q_{1}$ & 0.9150 \\
\hline Median & 1.4700 \\
\hline$Q_{3}$ & 2.8075 \\
\hline Standard Deviation & 1.0006 \\
\hline
\end{tabular}

To compare the transmuted two parameters Rayleigh distribution with base distribution we use criterions such as AIC, BIC and AICC. The better model or distributions have smaller AIC, AICC, and BIC.

$$
\begin{aligned}
& A I C=2 k-2 L \\
& A I C C=A I C+\frac{2 k(k+1)}{(n-k-1)} \\
& B I C=2 L+\operatorname{klog}(n)
\end{aligned}
$$

Where $k$ is the numbers of parameters in any distribution and $n$ is the sample size.

The results in Table 2 provide the least square estimation of transmuted two parameters Rayleigh, two parameters Rayleigh and one parameter Rayleigh distribution. This table also indicates the different information criterion values. In these results transmuted two parameters Rayleigh distribution has smallest AIC, AICC, and BIC as compared to one and two parameters Rayleigh distributions. This is employed that transmuted Rayleigh distribution is best fitted to precipitation data. Fig. 4 provides the graphical presentation of the empirical cdf compare with the transmuted two parameters Rayleigh distribution and its parent distribution. This figure also shows that transmuted two parameters Rayleigh distribution is better than its parent distribution.

Table 2: Least Square Estimation of Parameters and Goodness of Fit of the Transmuted Two Parameters Rayleigh Distribution and Related Distributions.

\begin{tabular}{lllll}
\hline Model & Estimates & AIC & AICC & BIC \\
\hline $\begin{array}{l}\text { Transmuted two pa- } \\
\text { rameters Rayleigh }\end{array}$ & $\begin{array}{l}\hat{\beta}=0.2270 \\
\hat{\alpha}=0.32\end{array}$ & 13.8225 & 14.2670 & 16.6249 \\
$\hat{\lambda}=0.4803$ & & & \\
$\begin{array}{l}\text { Two parameters Ray- } \\
\text { leigh } \quad(\lambda=0)\end{array}$ & $\hat{\beta}=9.6951$ & 19.0685 & 19.2113 & 20.4697 \\
$\begin{array}{l}\text { One parameter Ray- } \\
\text { leigh }(\mu=0, \lambda=0)\end{array}$ & $\hat{\alpha}=0.32$ & & & \\
\hline
\end{tabular}

\section{Ecdf of distances}

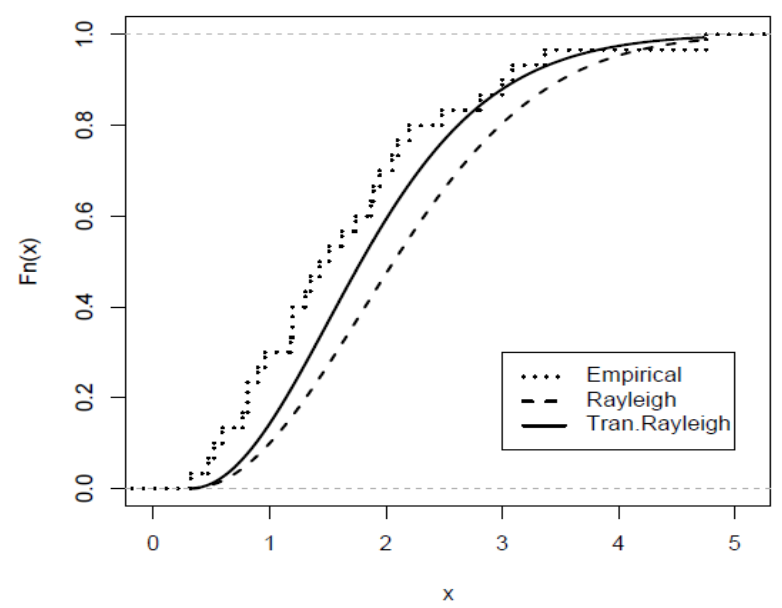

Fig. 4: Empirical Fitted Transmuted Two Parameters Rayleigh and Two Parameters Rayleigh cdf of the Precipitation Data Set.

\section{Conclusion}

The transmuted two parameters Rayleigh distribution introduced in this study. This generalized form of distribution has larger flexibility than base distribution. Plots of the density, distribution and reliability function have been sketched those shows the flexibility of proposed distribution. Some basic properties have been derived and the minimum, maximum, $r$ th order and joint order statistics are also studied. Least square estimation method is employed for the estimation of the parameters of the proposed distribution. To check the significance of this generalized distribution three goodness of fit criterion are used to select the most appropriate model. The comparison is done between three distributions such as one parameter Rayleigh, two parameters Rayleigh and transmuted two parameters Rayleigh distribution. Application of generalized distribution on a real data set is done that shows comparatively transmuted two parameters Rayleigh distribution is more appropriate than other types of the Rayleigh distributions. In conclusion we can say this new proposed distribution will help better in survival data analysis, precipitation and wind data. 


\section{Acknowledgements}

Authors are thankful to the editor and reviewers for their valuable comments to improve manuscript.

\section{References}

[1] Abd-Elfattah, A. M., Amal, S. H., \& Ziedan, D. M. (2006). Efficiency of Bayes estimator for Rayleigh distribution. Statistics on the Internet, 5.

[2] Dey, S., Dey, T., \& Kundu, D. (2014). Two-parameter Rayleigh distribution: different methods of estimation. American Journal of Mathematical and Management Sciences, 33(1), 55-74. http://dx.doi.org/10.1080/01966324.2013.878676.

[3] Dyer, D. D., \& Whisenand, C. W. (1973). Best linear unbiased estimator of the parameter of the Rayleigh distribution-Part I: Small sample theory for censored order statistics. Reliability, IEEE Transactions on, 22(1), 27-34. http://dx.doi.org/10.1109/TR.1973.5216019.

[4] Hyndman, R. J., \& Fan, Y. (1996). Sample quantiles in statistical packages. The American Statistician, 50(4), 361-365.

[5] Khan, H. M., Provost, S. B., \& Singh, A. (2010). Predictive inference from a two-parameter Rayleigh life model given a doubly censored sample. Communications in Statistics - Theory and Methods 39(7), 1237-1246. http://dx.doi.org/10.1080/03610920902871453.

[6] Leemis, L. M., \& McQueston, J. T. (2008). Univariate distribution relationships. The American Statistician, 62(1), 45-53. http://dx.doi.org/10.1198/000313008X270448.

[7] Merovci, F. (2013). Transmuted Rayleigh distribution. Austrian Journal of Statistics, 42(1), 21-31.

[8] Shaw, W. T., \& Buckley, I. R. (2009). The alchemy of probability distributions: beyond Gram-Charlier expansions and a skewkurtotic-normal distribution from a rank transmutation map. arXiv preprint arXiv:0901.0434.

[9] Tierney, L. (2009). Lisp-stat: an object-oriented environment for statistical computing and dynamic graphics (Vol. 353). John Wiley \& Sons.

[10] Lai, C. D. (2013). Constructions and applications of lifetime distributions. Applied Stochastic Models in Business and Industry, 29(2), 127-140. http://dx.doi.org/10.1002/asmb.948. 